\author{
Khvedelidze Mzia \\ Ivane Javakhishvili Tbilisi State University, Georgia \\ Jinjikhadze Levan \& Jinjikhadze Jemal \\ Sukhumi State University, Georgia
}

\title{
Integration of the Georgian Language and Mathematics in the Bilingual Teaching System
}

\begin{abstract}
Finding interdisciplinary links between language and mathematics attracts particular interest. Such interest is caused by the fact that the language is the basis of any perception process as the consciousness exists only within the language scopes and in the solving of any problem applying to the mathematic approach, mathematical thinking results in a new level of perception. In this respect, integration of the Georgian language and mathematics is of certain interest. Many issues of bilingual teaching in the didactic-methodic context can be solved very successfully on the basis of the integrated teaching of the Georgian language and mathematics. In our opinion, such integration, in the context of multilingual education, for the activation of the bilingual education process, is of great significance. The point is that integrated teaching of mathematics and the Georgian language clearly demonstrates common, general regularities of mathematics and Georgian and this, on the basis of simple observations and analysis, will help the student to find similar regularities in his/her native language and this is the main goal of bilingual teaching. Here observation, analysis and other methods of thinking are used naturally and not artificially, resulting in a natural character of the student's bilingual perception: isomorphism of relations between the mathematical and the Georgian language concepts are also revealed. Therefore, such integration can play a decisive role in the activation of bilingual teaching and this is the absolute guarantee of good results regarding the bilingual teaching.
\end{abstract}

Key words: multilingualism, bilingualism; teaching; Georgian language; mathematics; integration.

A great significance of the interdisciplinary methodology application in the teaching process is universally recognized. This concerns all subjects but identification of the inter-subject links between the language and mathematics deserves particular attention. This is because the language is the basis of any cognitive process. As consciousness exists only within the language and in the solving of a problem, a mathematical approach, relying on mathematical thinking, ensures a high level of cognition. In this respect, many issues of integration of the Georgian language and mathematics is of interest.

Primarily, we should argue that language is the means of communication. It is a certain code, a system of signs and a set of the rules for their use. The system includes the units of various levels: phonetic - sounds, intonation etc.; morphological - parts of the word: stem, prefix etc.; Lexical - words and their meanings; syntactic - sentences. Speech is an activity of humans, an individual psychological phenomenon, using the lingual code and system of signs. Speech is a language in action. In a speech, linguistic units, being in absolutely different interrelations with each other, create an unlimited number of combinations. Speech is always spread and arranged in time and it reflects the characteristics of the speaker and depends on the context and communication situations. The question is why do individuals need mathematical speech and mathematics in general? To become a mathematician? - Not at all! Mathematics and mathematical speech is necessary for all. Actual subjects, phenomena and processes in nature are seen from the point of view of mathematical thinking in an absolutely different way. 
Many issues of bilingual teaching, in didactical-methodological respect, can be perfectly resolved on the basis of integrated learning of the Georgian language and mathematics. In our opinion, such integration, in the multilingual education context, is of great significance for the activation of bilingual education. The integrated teaching of mathematics and Georgian language clearly demonstrates common, general regularities of mathematics and the Georgian language and this, on the basis of simple observations and analysis, will help the student to find similar regularities in his/her native language which is the main goal of the bilingual teaching. Here observation, analysis and other methods of thinking are used naturally and not artificially, resulting in a natural character of the student's bilingual perception, isomorphism of relations between the mathematical and Georgian language concepts is revealed. Therefore, such integration can play a decisive role in the activation of the bilingual teaching which is an absolute guarantee of good results in the bilingual teaching.

It should be noted that while discussing integration of mathematics and the Georgian language, we imply only general concepts of mathematics and Georgian, as only in their structural specificity can we find the isomorphism that, in our opinion, is the best tool for bilingual teaching perceived at the highest level. Such specific problems, as adding the fractions or solving equations and similar things which the students study at school and are subject to integration in Georgian language only artificially and therefore, cannot be effective in promoting the bilingual teaching. And the general issues that we consider in mathematics and Georgian have similar structural essence and order. These issues include:

$>$ Word, concept and term;

Language and speech;

$>$ Correlation between the concepts;

$>$ Euler-Venn diagram;

$>$ Operations on the sets;

> Separation of the sets into non-intersecting classes;

$>$ Classification of concepts;

Affixes;

Logical connections;

$>$ Quantifiers;

$>$ Binary relations;

$>$ Correspondence;

$>$ Exercises with empty spaces;

$>$ Discussion.

Let us discuss some of them.

Interrelations between the concepts are subject to similar laws in all disciplines, including Georgian language and mathematics. Understanding of the concept essence is impossible without understanding of its connection with other ones. Therefore, it is significant to know the relations between the concepts. We have to find out such relations each time we think, judge, consider, and for this, the best visual tool is the Euler - Venn diagram. For some reason, in the education literature, there is a widely spread opinion that there exist only these types of Euler-Venn diagrams (Fig. 1).

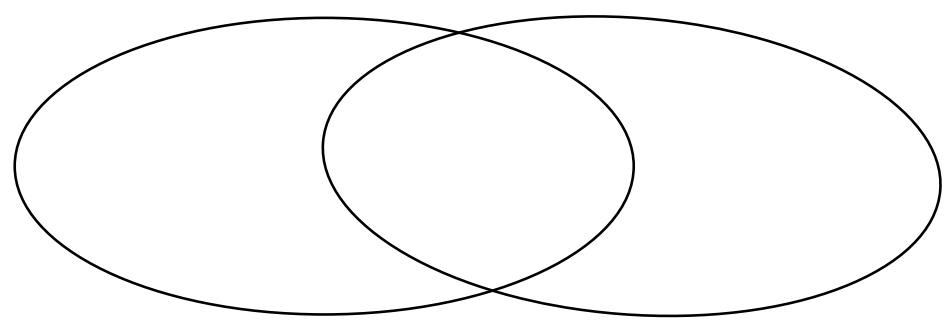

Fig. 1 
This is not correct, each figure below is a Euler-Venn diagram (Fig. 2):

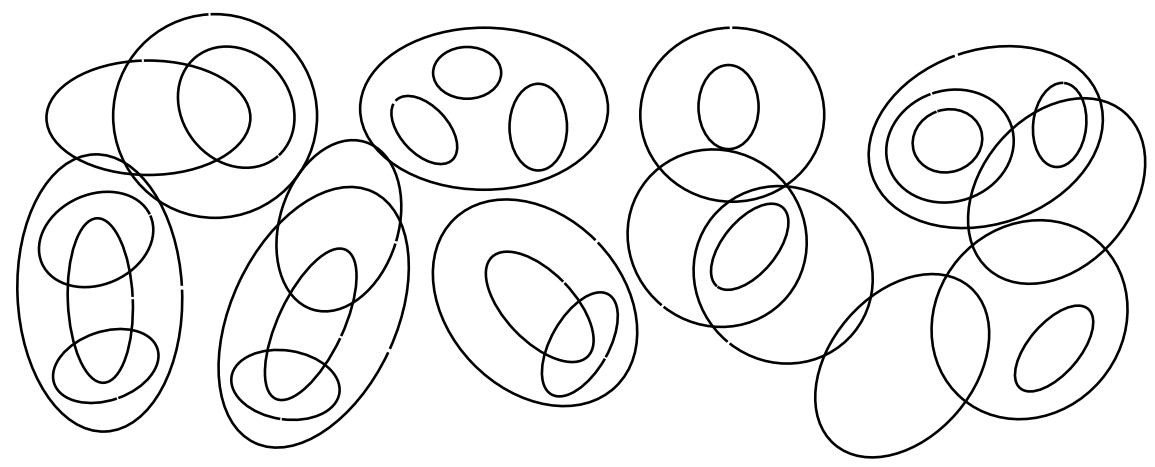

Fig. 2

Based on the above, it is clear that the following two types of exercises are useful in all disciplines:

1. Several concepts are given, interrelation between them is to be studied and Euler-Venn diagrams are to be built.

2. The concepts interrelations between which correspond to the given graphical schemes are to be found (Fig. 2).

We offer a few exercises.

Exercise 1. Euler-Venn's diagrams are given (Fig. 3):

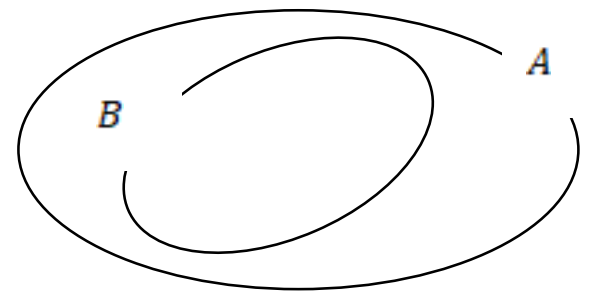

Fig. 3

What can be A and what can be B?

\section{Solution:}

Mathematics. A can be, for example, a set of triangles, B - a set of isosceles triangles, or A - a set of numbers, B - a set of natural numbers etc. An isosceles triangle is a type of a triangle, and a triangle is a class of isosceles triangle. Natural number is a type of number while the triangle is a class of isosceles triangle. This case is used in mathematics as the first basis for the definition of concepts. For example, the classes of isosceles triangle are polygons and geometric figures but the triangle is its closest class. Therefore, it is used in the definition of the isosceles triangle. In particular: the isosceles triangle is the triangle that ...etc.

The Georgian language. A can be, for example, the verb, B - an intransitive verb. A - a sentence, B - a simple sentence etc. The verb is the class of intransitive verbs while an intransitive verb is a type of the verb; a sentence is the class of simple sentence and simple sentence is a type of sentence. Certainly, in the first example, in the part of A, out of B, all verbs except for the intransitive ones should be placed. Similarly, in the second example.

Everyday situation. A can be, for example, the ball, B - a red ball. The ball is the class of the red ball, while the red ball is a type of a ball. Certainly, the part of A, which is out of B, should contain all the balls, except for the red ones. 
Independently compose and solve a similar exercise (both, in mathematics and Georgian) where the words are given and a Euler-Venn diagram is to be built!

Exercise 2. Euler-Venn diagrams are given (Fig.4):

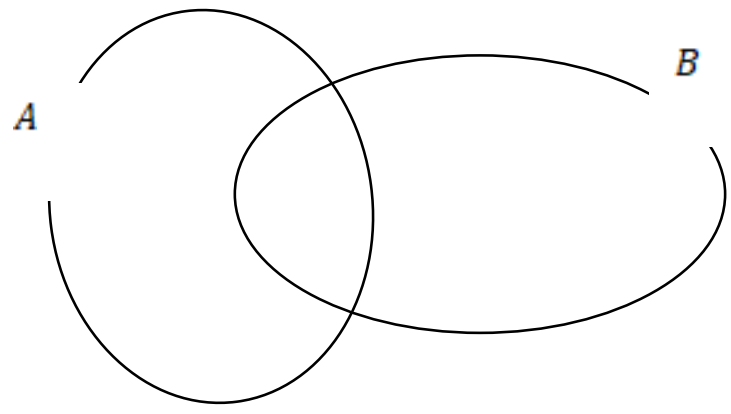

Fig. 4

Solution. View carefully the drawing: the circles (or closed curves) intersect. This means that if A is one concept and B - the other one, there is an item that belongs to both, A volume and B volume. It is not difficult to select the concepts for the drawing.

Mathematics. A) A - isosceles triangle, B - right-angled triangle. In the intersection there will be right isosceles triangles. In the part of A, out of the cross-section, there will be all isosceles triangles that are not right-angled etc. b) A - numbers that are divisible by $3, \mathrm{~B}$ - numbers divisible by 5 . The intersection will contain the numbers that are divisible by both, 3 and 5, i.e. those that are divisible by $15(15,30,45,60 \ldots)$ etc.

The Georgian language. A - inanimate nouns, B - specific nouns. The cross-section will contain all inanimate specific nous. In the part of $A$ that is out of the intersection, all inanimate abstract nouns will be placed while the part of $\mathrm{B}$, out of intersection, will contain animate specific nouns.

Everyday situations. A - a rubber ball, B -a red ball. In the intersection there will be a red, rubber ball. The part of A, out of intersection, will contain all balls except for those, that are red and in the part of B, out of intersection, there will be all red balls that are not made of rubber.

Exercise 3. The following concepts are given: root- syncopating nouns and family nouns. Compose the corresponding Euler-Venn diagrams.

Solution. View carefully the word connections, you will notice that the nouns that are non-rood syncopating, are out of the first connection and all nouns except for the family ones are left out of the second connection. Some family nouns are root-syncopating whilst some are not. Some of the root-syncopating nouns are the family ones while some of them are not. Hence, these two connections of words, more exactly, their meanings, have common elements, i.e. there are the root-syncopating family nouns and they also have noncommon specific elements, i.e. the Euler-Venn diagrams of the given word connections (concepts) will be Fig. 4: A - root-syncopating nouns, B - family nouns.

Intersection contains root-syncopating family nouns. In the part of $\mathrm{A}$, out of intersection, there are the rootsyncopating non-family nouns and in the part of B, out of intersection, there are the root-syncopating family nouns.

Exercise 4. Given: words, nouns, adjectives. Analyze interrelations between them and compose respective Euler-Venn's diagrams.

Solution. No complicated considerations are required to make a conclusion that the corresponding EulerVenn diagrams are constructed as follows: 


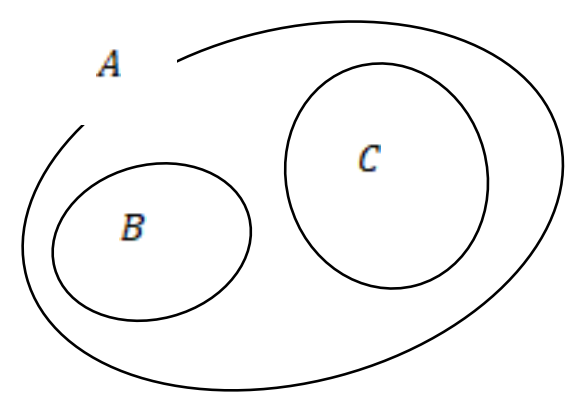

Fig. 5

Exercise 5. Euler-Venn's diagrams are given (Fig.6):

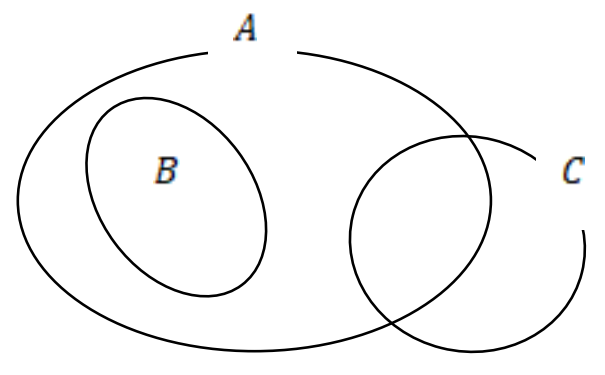

Fig. 6

Select the concepts according to the given diagrams.

Mathematics. A B concept is required that has all elements common with A, though not filling A. In addition, the $\mathrm{C}$ concept should be found that has common elements with $\mathrm{A}$ and has nothing in common with $\mathrm{B}$. Such are, for example: A - integers, B - natural numbers, C - negative numbers. In the part of A, out of the intersection with $\mathrm{B}$ and $\mathrm{C}$, there will be only zero and in the intersection of $\mathrm{A}$ and $\mathrm{C}$, there will be the negative integers. In the part of $\mathrm{C}$ out of $\mathrm{A}$, there will be all negative numbers, with the exclusion of negative integers.

The Georgian language: a) A -a ball, B - a non-rubber red ball, C - rubber toys. In the intersection of A and $\mathrm{C}$, there will be a rubber ball, in the part of $\mathrm{C}$, out of $\mathrm{A}$, there are all the rubber toys except for the balls. In the part of $\mathrm{A}$ out of intersection with $\mathrm{B}$ and $\mathrm{C}$, there will be all balls that are not red and are not made of rubber. b) A - root-syncopating nouns, $\mathrm{B}$ - root-syncopating geographical names, $\mathrm{C}$ - eliding stem nouns.

Certainly, in the intersection of $\mathrm{A}$ and $\mathrm{C}$, there will be the nouns that are root-syncopating and stem

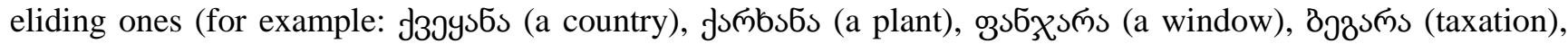

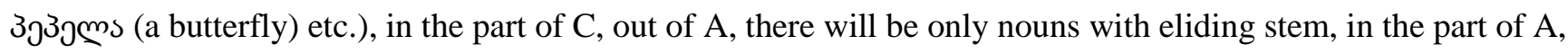
out of intersection with $\mathrm{B}$ and $\mathrm{C}$, there will be the root-syncopating non-geographical nouns.

Exercise 6. Given: A - connecting conjunctions, B - grouping conjunctions, $\mathrm{C}$ - composed conjunctions. Analyze their correlation and compose respective Euler-Venn diagrams.

Solution. As a result of analysis of correlations between the given concepts we make the conclusion that the grouping conjunction is a type of connecting conjunction. Therefore, set B is entirely included into A. It has nothing common with $\mathrm{C}$, as grouping conjunctions ("and", "or") are simple. A and $\mathrm{C}$ have both, common and different elements. Hence, the relevant Euler-Venn diagram will be: Fig. 6.

Exercise 7. Euler-Venn diagrams are given (Fig.7): 


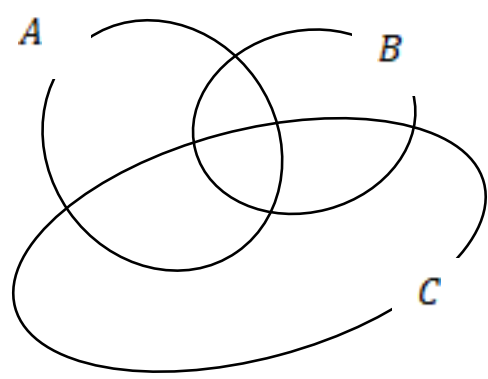

Fig. 7

Select the concepts in accordance with the given diagrams.

Solution. From the diagrams it is clear that all three concepts should have the intersections by pairs and in addition, all three concepts should have the common elements.

Mathematics. A - natural numbers multiples of 2, B - natural numbers multiples of 3, C-natural numbers multiples of 5. In the intersection of $A$ with $B$, there will be the numbers divisible by 6 , in intersection of $A$ with $\mathrm{C}$, there will be numbers divisible by 10 and in the intersection between $\mathrm{B}$ and $\mathrm{C}$ there will be the numbers divisible by 15 . In the intersection of all three sets there will be the numbers divisible by 30 . Substance of the other areas of the diagram is clear as well.

The Georgian language. A - words with the stems which end in vowels, B - words with full stems, C words with simple stems. We know that there are the words with full stems which end in vowels, there are also the words with simple stems which end in vowels and there are the words with full and simple stems. In addition, there are words that have full simple stems ending in vowels. Hence, it is easy to see which words are placed in which areas of the diagram.

Exercise 8. Given: A - wooden things, B - round things, C - tables. Analyze correlation between them and compose relevant Euler-Venn diagrams.

Solution. As there exist a round wooden thing, a wooden table and a round table, A, B and C pairs have common elements, i.e. pairs of these sets are intersecting and their relevant concepts are compatible in pairs. Existence of a round wooden table means that there are the common elements of all three sets as well. Hence, the sought diagrams are as per Fig. 7.

Knowledge of the correlations between the concepts and use of Euler-Venn diagram is very helpful for designing logical activities for the elementary school students and solving of the problems intended for testing of general skills.

Dividing of the set into the classes that do not intersect both, in the Georgian language and mathematics is a very significant operation. Let us examine this issue using the examples.

\section{Mathematics.}

Exercise 9. A set of numbers is given $\{12,15,35,36,60,288,355,405,1272$, and 1293$\}$

1. Apply to it two characteristics: divisibility by 3 and divisibility by 5 !

2. What classes of numbers are obtained?

3. Are these classes non-intersecting? Why?

4. Distribute the given numbers in Euler-Venn diagrams!

Solution. As a result of distribution of these numbers in an Euler-Venn diagram based on the given characteristics, the given set of numbers was divided into three non-intersecting classes; i.e. given that $\mathrm{X}$ is the set of multiples of 3 and $\mathrm{Y}$ - set of multiples of 5, we obtain three non-intersecting areas on the diagrams (Fig. 8):

A - numbers that are multiples of 3 and are not multiples of 5 .

$\mathrm{B}$ - numbers that are multiples of 5 and are not multiples of 3 .

$\mathrm{C}-$ numbers that are multiples of both, 3 and 5 . 


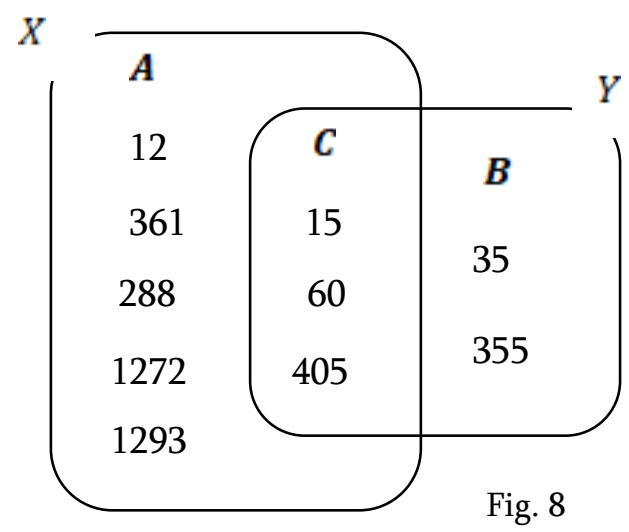

Classes obtained as a result of division are non-intersecting as none of the pairs have common elements.

\section{The Georgian language}

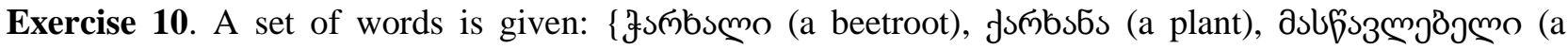

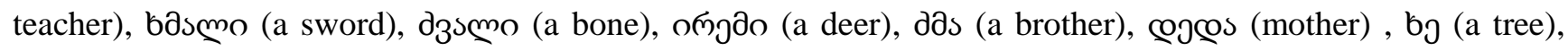

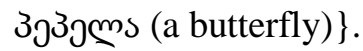

1. Apply to it two characteristics: root-syncopating and eliding stem!

2. What classes of words are obtained?

3. Are these classes non-intersecting? Why?

Solution. As a result of distribution of the given words in an Euler-Venn diagram based on the given characteristics the given set of words was divided into three non-intersecting classes, i.e. assuming that $\mathrm{X}$ is the set of eliding stem words and $\mathrm{Y}$ - set of root-syncopating words, on the diagram we shall have three nonintersecting areas (Fig. 9):

A - words that have eliding stem and are not root-syncopating.

$\mathrm{B}$ - words that are root-syncopating and have no eliding stem.

$\mathrm{C}$ - words that are root syncopating and have eliding stem.

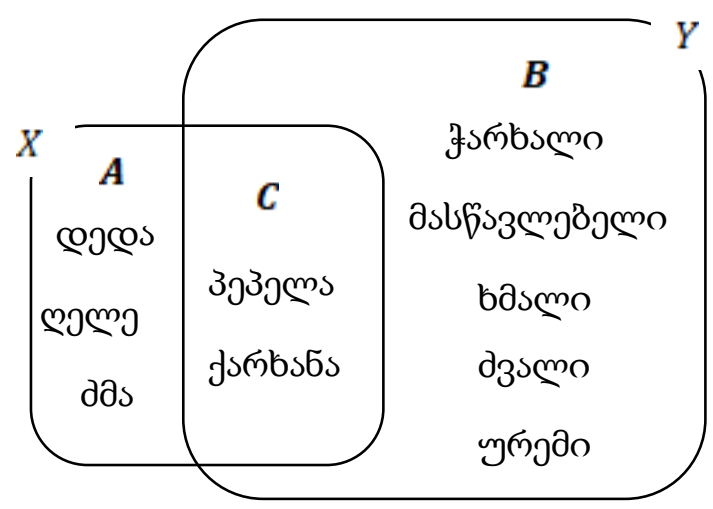

Fig. 9

Classes obtained as a result of dividing are non-intersecting as none of the pairs have common elements.

\section{Logical problems}

Problem 1. All children in the class study English and French languages. 17 of them study English language and 15 - French. And 8 children study both languages simultaneously. How many children are in the class? 
Solution. Discussion is as follows: let us find the number of children studying English and French languages. These will be $17+15=32$ children. According to the statement of the problem, 8 children study both languages simultaneously. And hence, we can calculate number of children in the class. For this, from 32 children, studying English and French languages, we have to deduct 8 children studying both languages simultaneously as this number was counted twice: $32-8=24$.

If we have drawn Euler-Vann diagrams, the problem would be solved very easily.

Answer: there are 24 students in the class.

Problem 2. Of 100 students, 31 study English language, 29 - German, 44 - French, 6 English and German, 9 - English and French, 5 - German and French and 4 students study all three languages. What is the number of students who do not study any language?

Solution by discussion, similar to the previous problem, is quite difficult and hence, it is easy to make mistakes. Therefore, let us solve this problem directly using the Euler-Venn's diagrams (Fig. 10).

Solution. Certainly, the given set of 100 students is divided into non-intersecting classes (sub-sets) based on three characteristics, as the number of languages is three. At the same time, these three sets are intersecting, i.e. Euler-Venn diagrams will be as follows (Fig. 10A):
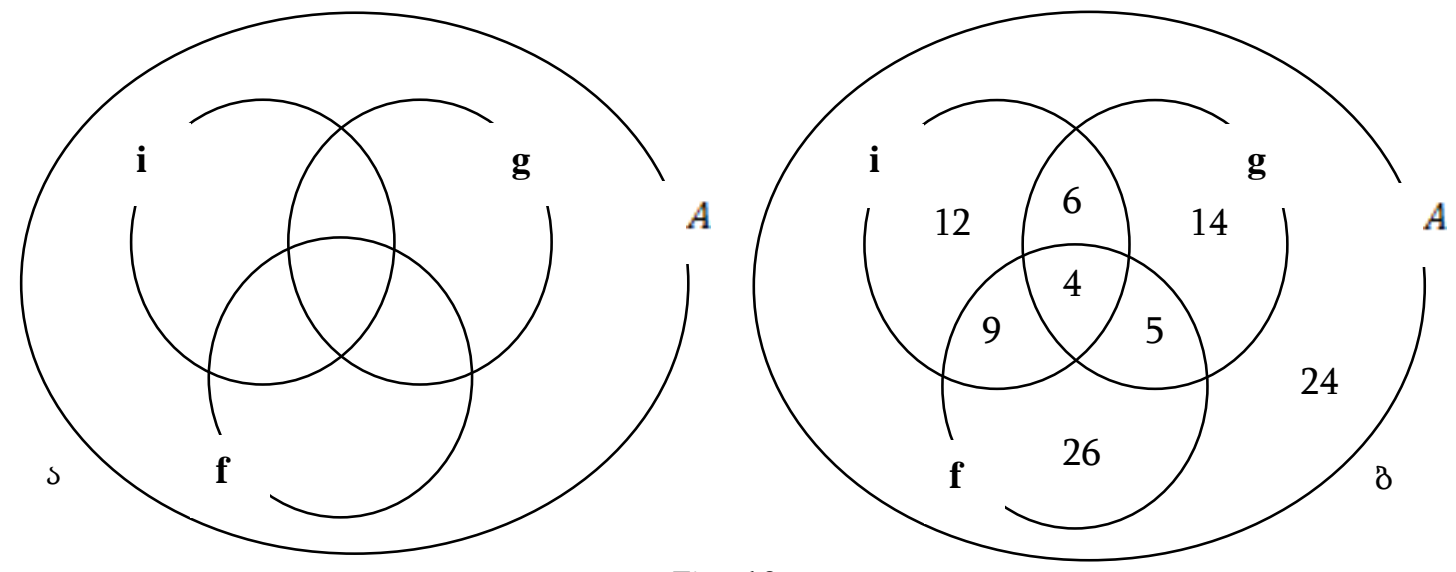

Fig. 10

Where $\mathrm{A}$ is the given set of 100 students and $\mathbf{i}, \mathbf{g}$ and $\mathbf{f}$ are, respectively, English, German and French languages. Let us include into the diagrams the known numbers of students learning by two and three languages: 6, 9, 5 and 4 (Fig. 10B). Let us calculate the numbers of students studying one language: 12, 14, 26 (these are obtained as follows: $12=31-(6+9+4) ; 14=29-(6+5+4) ; 26=44-(9+5+4))$ and place these results into the diagrams. Further, we shall easily find the number of students not studying any language:

$100-(12+14+26+9+5+6+4)=100-76=24$.

Answer: 24 students do not learn any of the languages.

We should also note use of the concept of correspondence of the sets.

Exercise 11. A and B word sets are given (Fig. 11). Draw the arrows from the elements of the first set drawn as points to the elements of the other so that the correspondence was found between the sets A and B:

In the Georgian language: based on similarity of stems.

In mathematics: based on equality relation.

\section{Solution.}

(The Georgian language) based on morphological analysis of the words and seeking of the words with similar stems, the correspondence graph will be as follows (Fig. 11): 


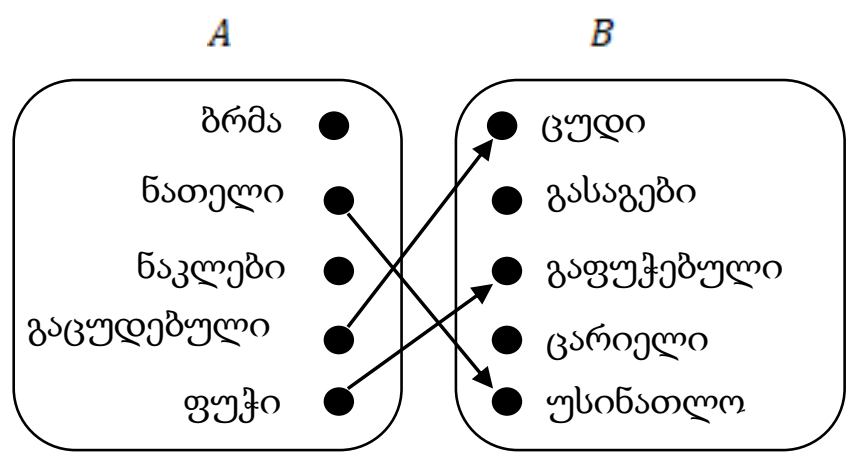

Fig. 11

(Mathematics) if we calculate the expressions, the graph of correspondence will be as follows (Fig. 12):

A

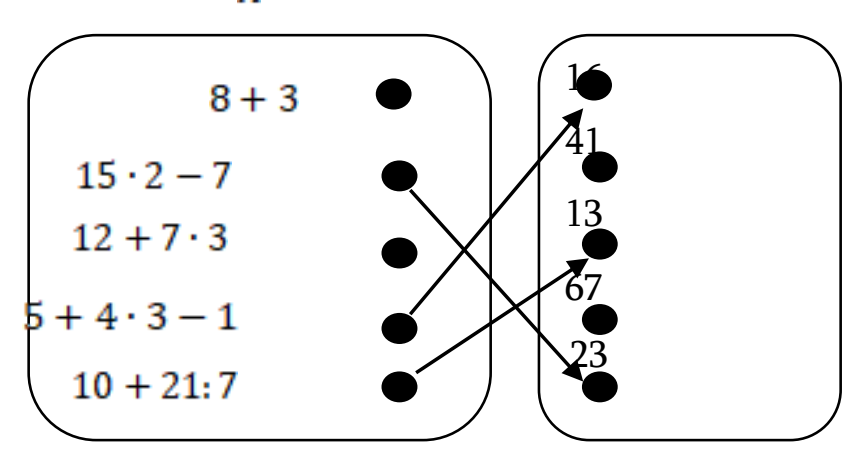

Fig. 12

Exercises with empty spaces are used in all disciplines but in mathematics they are of particular significance as they serve to numerous goals, among which the main goal is the development of a functional idea. In mathematics, the empty spaces are in the equations, in equations and, generally, in predicates, sometimes these are simply unknown values and sometimes - the variables. Such exercises play a particular role in the teaching of the Georgian language as well.

In the Georgian language a word can be taken separately, unlike mathematics, as a word has the meaning and any letter cannot be substituted into the empty space. For example:

Exercise 12. Fill in the empty spaces to obtain words:

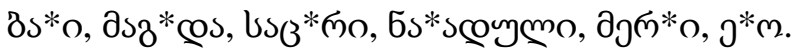

Exercise 13. Fill in the empty spaces to obtain words:

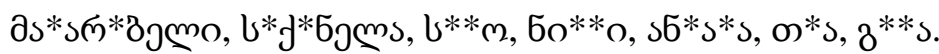

In such exercises the results can differ. For example, the first word of the first exercise can be " 8 sळo" for one student and " 3560 " - for the other etc. The fourth word of the second exercise can be interpreted by one student as "6oszo" and by the other - as "Бомळo". The fifth word of the same exercise can be "s6cos $8 s$ " for one student and "s6zsms" - for the other etc.

This is to be welcomed.

The empty spaces may imply the words or even sentences. For example:

Exercise 14. Examine carefully the given words and fill the empty spaces. 

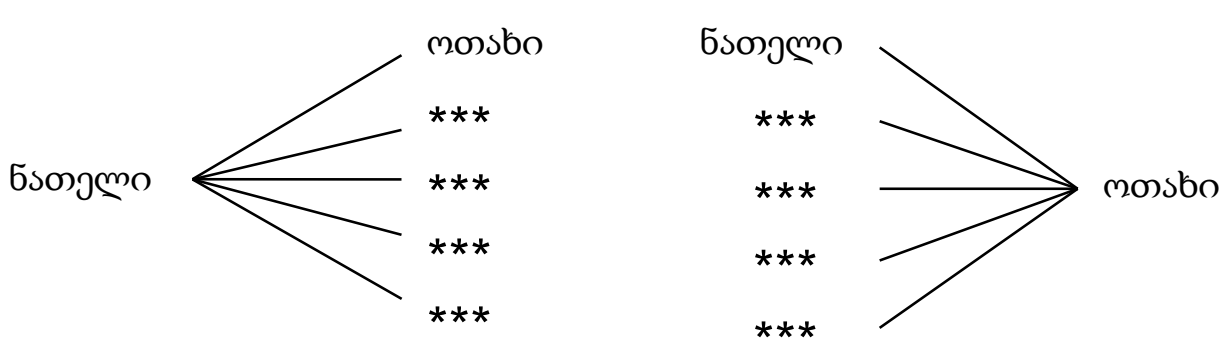

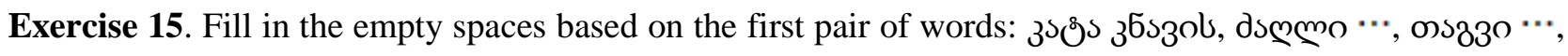

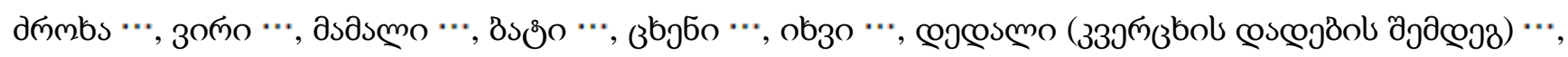

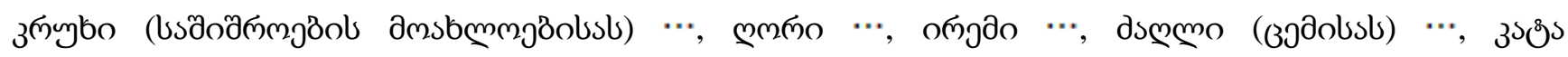

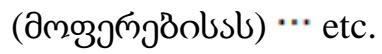

It seems that integration of mathematics and the Georgian language takes place on the basis of their interdisciplinary links and it occurs against the background of understanding of the isomorphism of general internal regularities of these two disciplines. Therefore, such integration can play a decisive role in the activation of bilingual teaching.

\section{References}

Gabunia, 2010 - Kakha Gabunia, Specificities of Content and Language Integrated Teaching in Bilingual Classroom Setting: Journal Bilingual Education, \#1, 2010. Tbilisi.

Mehisto, Frigols, Marsh, 2008 - Peeter Mehisto and Maria Jesus Frigols and David Marsh, Uncovering CLIL; Macmillan Books for Teachers, 2008.

Coyle, Hood, Marsh, 2010 - Do Coyle, Philip Hood, David Marsh, CLIL (Content and Language Integrated Learning); Cambridge, 2010. 